\title{
Avaliações clínica e morfométrica da capacidade angiogênica da membrana de quitosana em córneas de coelhos
}

\author{
Clinical and morphometric evaluations of the angiogenic capacity \\ of chitosan membrane in rabbitcorneas
}

\author{
Thiago André Carreo Costa ${ }^{1}$ \\ Alexandre Lima de Andrade ${ }^{2}$ \\ Thaís Eliane Binotto ${ }^{3}$ \\ Ana Maria de Guzzi Plepis ${ }^{4}$ \\ Lilian Bevilacqua ${ }^{5}$ \\ Wilson Machado de Souza ${ }^{6}$
}

\section{RESUMO}

Objetivo: Estudar a vascularização corneal (VC) induzida pela membrana de quitosana (MQ) implantada por enxertia interlamelar na córnea de coelhos. Métodos: Foram utilizados 16 coelhos. No olho esquerdo procedeu-se enxertia interlamelar de um fragmento de $0,25 \times 0,25 \mathrm{~cm}$ de MQ (olho tratado). No olho direito realizou-se apenas a microbolsa estromal (olho controle). Avaliaram-se clinicamente os animais aos 1, 3, 7, 15 e 30 dias de pós-operatório. Aos 30 dias mensurou-se a VC pelo Sistema Analisador de Imagens LEICA QWIN-550 ${ }^{\circledR}$. Resultados: Aos sete dias observou-se VC a 1,5 $\pm 0,93 \mathrm{~mm}$ do limbo em direção ao eixo visual. Aos 15 dias houve aumento da VC $(4,75 \pm 3,20 \mathrm{~mm})$, que se manteve aos 30 dias $(4,25 \pm 4,10 \mathrm{~mm})$. Os olhos controles não apresentaram quaisquer alterações oculares. Houve diferença estatística $(\mathrm{p}<0,05)$ entre as áreas corneais vascularizadas dos olhos tratados e controles aos 15 e 30 dias de pós-operatório. Conclusões: A MQ induziu angiogênese corneal quando aplicada à córnea de coelhos por enxertia interlamelar, a qual persistiu de forma leve até 30 dias de pós-operatório. Embora estudos adicionais sejam necessários a MQ poderá ser mais uma opção de membrana para enxertos em ceratoplastias.

Descritores: Membranas artificiais; Córnea; Quitosana; Coelhos

\section{INTRODUÇÃO}

A córnea, que representa uma das estruturas da túnica fibrosa do bulbo ocular, constitui-se em tecido vulnerável, dada a sua localização externa. Ela é responsável primariamente pela visão, e tem sido estudada intensivamente no homem e animais nos últimos tempos, devido ao crescente número de doenças corneais, algumas ainda, de etiologia e tratamento desconhecidos ${ }^{(1)}$.

Quanto às doenças corneais, há que se considerar que não se trata de matéria que dispensa novas e originais investigações, sobretudo o uso de membranas biológicas na reparação da córnea com importante perda tecidual. No homem, grande parte dos enxertos corneais não se trata de tecidos xenólogos ou heterólogos preservados ${ }^{(2)}$. No entanto estes, quando utilizados, são capazes de induzir a vascularização ${ }^{(3-4)}$. Deste fenômeno, é possível ocorrer a pigmentação corneal tardia, rejeição do tecido implantado, fibrose e leucomas cicatriciais, que resultariam em sucesso parcial, constituindo-se, assim, em ceratoplastias tectônicas ${ }^{(5)}$.

A quitosana é um polissacarídeo linear abundante, derivado da N-desa- 
cetilação da quitina e composto principalmente de h-(1-4) ligado a 2-desoxi-2-amino-d-glicopiranose e parcialmente de h-(1-4) ligado e 2-desoxi-2-acetamido-glicopiranose. Trata-se de um polissacarídeo abundante na natureza, constituindo-se em uma fonte renovável e relativamente barata uma vez que é obtida, também, como material rejeitado pela indústria da pesca ${ }^{(6-8)}$. Naturalmente, a quitosana é encontrada em alguns fungos, mas é principalmente obtida do exoesqueleto de crustáceos como camarões, caranguejos e moluscos como as lulas ${ }^{(6)}$. Devido à sua biocompatibilidade, natureza não tóxica e propriedades como efeitos inibitórios no crescimento de fungos e bactérias, há um interesse crescente em seu potencial na aplicação em enxertos ${ }^{(9-11)}$. Estudos recentes têm focado seus interesses nas atividades anti-angiogênicas da quitosana ${ }^{(11)}$. A utilização da membrana de quitosana (MQ) em ceratoplastias ainda não fora descrita, nem mesmo sua capacidade de induzir a vascularização corneal (VC), motivando-nos a realizar esta pesquisa, no sentido de saber da sua viabilidade de aplicação em cirurgias corneais.

O objetivo deste trabalho foi estudar os aspectos clínicos, bem como, a capacidade angiogênica da MQ implantada em microbolsa no estroma corneal de coelhos, por meio da mensuração da quantidade de vasos neoformados na área total da córnea.

\section{MÉTODOS}

\section{Animais}

Foram utilizados 16 coelhos, da raça Nova Zelândia, fêmeas, com peso médio de $3,0 \mathrm{~kg}$. Os animais foram fornecidos pelo Biotério Central da Faculdade de Odontologia de Araçatuba - Unesp. Foram mantidos em gaiolas suspensas, com água e ração “ad libidum”. Previamente à realização das manobras de enxertia interlamelar da MQ, os animais foram submetidos a exame oftálmico, onde se buscou a presença ou não de secreção ocular, blefarospasmo/fotofobia, hiperemia conjuntival, opacidade corneal, vascularização corneal e defeito epitelial (analisado pelo teste de fluoresceína).

\section{Grupos experimentais}

Constituíram-se dois grupos experimentais (G1 e G2). Nos olhos esquerdos de cada animal, após confecção da microbolsa estromal, realizou-se enxertia interlamelar da MQ, constituindo assim, o grupo tratado (G1). Nos olhos direitos foram realizadas apenas as microbolsas estromais, constituindo o grupo controle $(\mathrm{G} 2)$.

\section{Membrana de quitosana}

A membrana de quitosana utilizada no presente estudo foi confeccionada e fornecida pelo Laboratório de Bioquímica e Biomateriais do Instituto de Química da USP - São Carlos.

\section{Procedimento cirúrgico}

Os animais foram anestesiados por anestesia dissociativa com quetamina $(35 \mathrm{mg} / \mathrm{kg})$ e xilazina $(5 \mathrm{mg} / \mathrm{kg})$ aplicados por via intramuscular em mesma seringa.
Após preparação do campo operatório, com auxílio do equipamento para esteroscopia (microscópio estereoscópico modelo Leica MZAPO ${ }^{\circledR}$ ), foi realizada ceratotomia no olho esquerdo de cada animal, com incisão corneal realizada próximo ao limbo (1,0 mm do mesmo). Com auxílio de uma espátula microcirúrgica, o estroma foi divulsionado para confecção da microbolsa na porção média da espessura estromal.

Nos olhos dos animais G1, fragmentos de $0,25 \times 0,25 \mathrm{~cm}$ de MQ foram introduzidos no fundo da microbolsa corneal, que teve seu limite na região axial da córnea. Nos olhos direitos foi confeccionada somente a microbolsa corneal sem enxertia da MQ (G2). A figura 1 representa esquematicamente a enxertia interlamelar da MQ.

\section{Pós-operatório}

Como procedimentos pós-operatórios, os animais receberam pomada antibiótica (Epitezan ${ }^{\circledR}$, Frumtost) a intervalos de 24 horas, durante 10 dias. Para minimizar o desconforto ocular, foi utilizado buprenorfina $\left(\right.$ Tengesic $^{\circledR}$ ) na dose de $0,05 \mathrm{mg} / \mathrm{kg}$, pela via subcutânea a cada 12 horas, durante 7 dias.

\section{Avaliação clínica}

Os olhos operados foram avaliados aos 1, 3, 7, 15 e 30 dias de pós-operatório na busca dos fenômenos intercorrentes com a evolução clínico-cirúrgica e demais manifestações ocu$\operatorname{lares}^{(12-15)}$. Os eventos pesquisados foram os mesmos adotados por ocasião da avaliação clínica pré-cirúrgica. Cada animal teve sua ficha clínica individual onde foram computados os eventos oculares observados por dois examinadores que não tinham conhecimento prévio dos grupos.

\section{Avaliação morfométrica da angiogênese}

Foi realizado estudo morfométrico em ambas as córneas de cada animal ao final de 30 dias de pós-operatório. Após nova anestesia, segundo os procedimentos adotados por ocasião das ceratotomias, os animais foram submetidos a exame morfométrico de ambas as córneas para mensuração da angiogênese induzida pela MQ e pelo trauma cirúrgico resultante da confecção da microbolsa. Para tanto, foi utilizado o Sistema Analisador de Imagens LEICA QWIN 550 ${ }^{\circledR}$ que registrou as áreas totais e áreas vascularizadas das córneas. O sistema analisador de imagem foi mantido a uma distância de $50 \mathrm{~cm}$ da superfície da córnea de todos os animais. Ainda, foi avaliada a distância dos vasos corneais do limbo até o eixo axial da córnea, adotando os seguintes parâmetros: ausência (-), (2 mm) - $2 \mathrm{~mm}$ do limbo; (4 mm) - 4 mm do limbo; (6 mm) - 6 mm do limbo; (ev) vasos no eixo visual ${ }^{(16)}$.

\section{Análise estatística}

Para a variável distância dos vasos do limbo em direção ao enxerto foi realizado o teste Tukey. Para a análise da área vascularizada absoluta, e relativa à área total da córnea, utilizou-se o Teste t student para comparar tais variáveis entre os dois grupos. As diferenças estatísticas foram consideradas significantes 


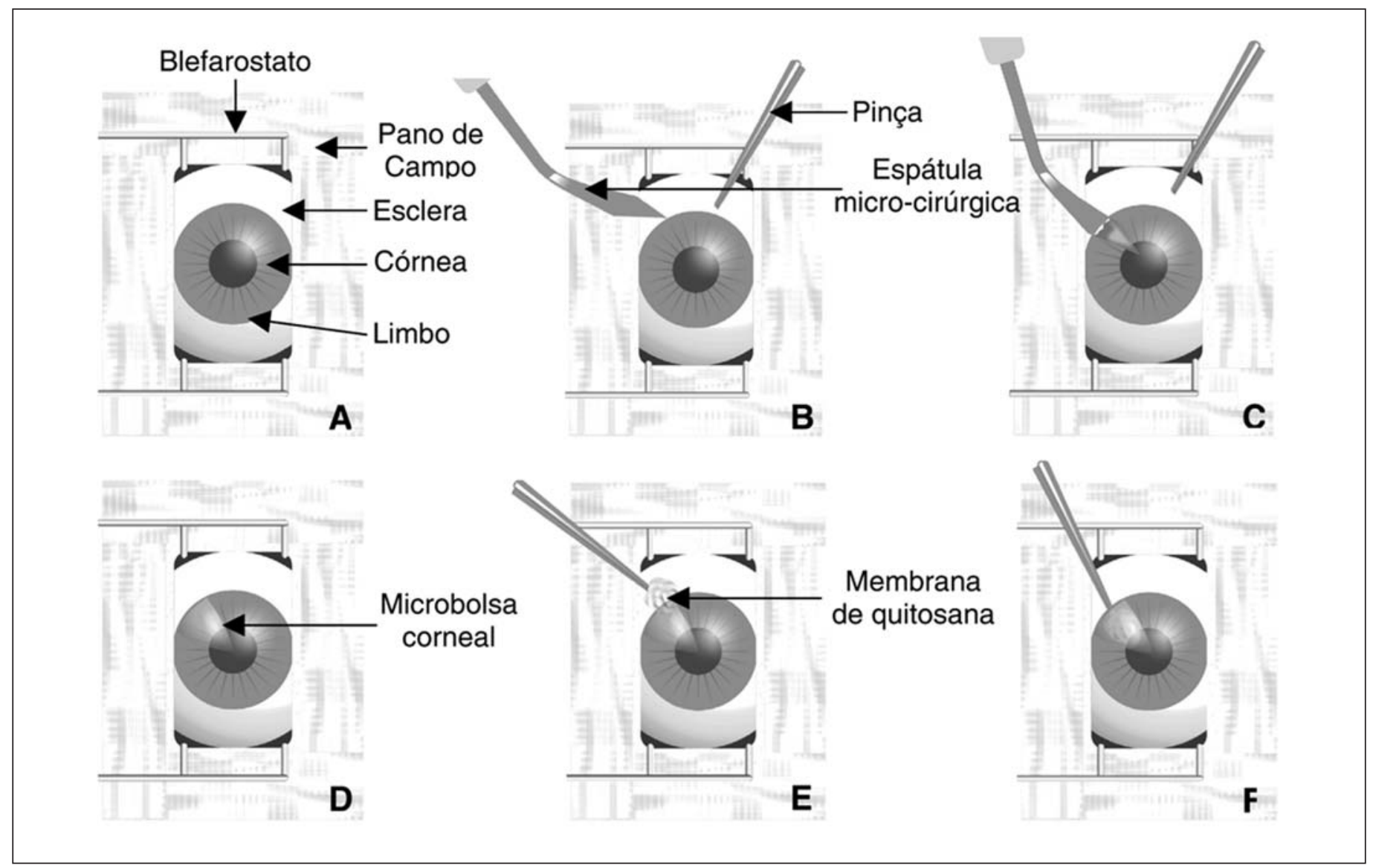

Figura 1 - Esquema ilustrativo do procedimento cirúrgico de enxertia interlamelar da MQ. Em A, campo cirúrgico preparado. Em B, delimitação do local da incisão da córnea para confecção da microbolsa (limbo) com a ponta da espátula micro-cirúrgica. Em C, confecção da microbolsa corneal. Em D, microbolsa corneal concluída. Em E, início da enxertia da MQ. Em F, enxertia interlamelar da membrana de quitosana.

quando $\mathrm{p}<0,05$. As análises estatísticas foram efetuadas empregando-se o programa SAS (Statistical Analysis System) ${ }^{(17)}$.

\section{RESULTADOS}

O estudo clínico possibilitou observar a evolução pósoperatória dentro dos padrões normais compatíveis com ceratoplastias interlamelares com tecidos xenólogos ${ }^{(3-5)}$. A evolução clínica oftálmica variou entre os grupos estudados. Os olhos direitos de todos os animais, não apresentaram quaisquer alterações clínicas.

A maioria dos olhos $(n=14)$ que receberam enxerto de MQ apresentou secreção ocular discreta a partir do terceiro dia de pós-operatório. Este fenômeno tornou-se ausente nos períodos subseqüentes. Blefarospasmo, fotofobia e hiperemia conjuntival manifestaram-se de forma moderada a grave, nos três primeiros dias de pós-operatório. Entre o $7^{\circ}$ e $15^{\circ}$ dias de observação oito olhos tratados apresentaram leucoma corneal que regrediu ao final da observação. O teste de fluoresceína para avaliação de defeito epitelial foi negativo em todos os momentos, em todos os olhos avaliados. Não foram observados sinais clínicos de rejeição do implante tais como perda do enxerto, ruptura da microbolsa, abscesso e necrose corneais em nenhum dos olhos que receberam enxerto de MQ.

Do ponto de vista morfométrico, na análise da $\mathrm{VC}$ aos 30 dias de pós-operatório, observou-se diferença estatística significante entre os grupos estudados. Nos olhos esquerdos (G1) a VC foi observada já ao terceiro dia de pós-operatório e caracterizava-se por aumento da vascularização límbica em forma de escova (brush like). Tais vasos foram claramente observados aos sete dias e distavam do limbo em média 1,5 $\pm 0,93 \mathrm{~mm}$. Aos 15 dias de pós-operatório, observou-se aumento na vascularização corneal, cujos vasos encontravam-se a 4,75 $\pm 3,2 \mathrm{~mm}$ do limbo. Aos 30 dias, os vasos estavam distantes do limbo 4,25 44,1 mm. A análise estatística indicou que as médias encontradas aos 15 e 30 dias de pós-operatório não diferiram entre si, enquanto, tais médias diferiram estatisticamente $(\mathrm{p}<0,05)$ das médias encontradas aos 1, 3 e 7 dias de observação. As médias do G1 (tratado) comparadas com as do G2 (controle) apresentaram diferença estatística no $15^{\circ}$ e $30^{\circ}$ dias.

Os valores obtidos da área total da córnea dos olhos tratados (G1) aos 30 dias foram em média, $1,47 \pm 0,16 \mathrm{~cm}^{2}$, enquanto

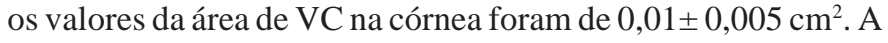
razão entre a área de vasos na córnea e a área total da córnea 
teve uma média de $0,88 \pm 0,39 \%$. A figura 2 ilustra os olhos do G1 e G2 aos 30 dias de pós-operatório.

\section{DISCUSSÃO}

A técnica de indução de vascularização na córnea de coelhos foi uma das primeiras a serem desenvolvidas ${ }^{(18)}$. Nos últimos 50 anos, coelhos, ratos e cobaias vêm sendo utilizados extensivamente como modelo experimental de rejeição ao enxerto corneal imunomediada. A principal característica anatômica e biológica que determina o privilégio imunológico da córnea é o fato de a mesma ser avascular. Estudos mostram que a antigenicidade das células corneais é menos significante quando comparada às células de outros tecidos no organismo ${ }^{(19)}$.

O trauma cirúrgico da enxertia compromete o privilégio imunológico da córnea, colocando o sucesso da enxertia em risco, podendo levar à rejeição imunomediada. A rejeição ocorrida quando da utilização de tecidos xenólogos não é mediada por células, mas sim pelo complexo maior de histocompatibilidade $^{(19)}$. Vários métodos têm sido desenvolvidos para estudar a angiogênese, pois conhecendo como controlála, há grande probabilidade de se evitar muitas das maiores causas de perda visual ${ }^{(20)}$.

Optou-se em estudar a capacidade de indução de VC da $\mathrm{MQ}$, primeiramente pelo fato de tratar-se de um biomaterial original, aliado ao fato de não haver relatos do seu uso em oftalmologia. Ainda, porque se busca um material biocompatível, eficaz na reparação de lesões corneais e de baixos custos.

A córnea se comportou como um bom modelo experimental para estudo da angiogênese, pois, além de ser transparente e avascular, permitiu a mensuração quantitativa do crescimento vascular a partir do limbo ${ }^{(21)}$. O protocolo anestésico adotado neste experimento promoveu boa imobilização e analgesia do animal, permitindo a execução do procedimento proposto com tempo de duração e segurança adequados.
A angiogênese está presente em inúmeros processos fisiológicos ou patológicos. Entendê-la e saber controlá-la resulta na obtenção de um tratamento efetivo para doenças angiogênicas, retinopatia diabética, fibroplasia retrolentiniana e rejeição de órgãos ou tecidos transplantados ${ }^{(18,22)}$. Clinicamente, pôdese verificar que a MQ induziu a VC já a partir do $3^{\circ}$ dia de pósoperatório, tornando-se intensa aos 15 dias e regressa ao final da observação. Tal fenômeno deve-se exclusivamente à presença da membrana na córnea, uma vez que, as córneas controles não apresentaram quaisquer alterações. No que se refere à distância dos vasos a partir do limbo em direção aos enxertos, verificou-se que as médias diferiram estatisticamente aos 15 e 30 dias de pós-operatório em relação aos olhos controles (G2).

O Sistema Analisador de Imagens LEICA QWIN $550^{\circledR}$ utilizado para avaliação da $\mathrm{VC}$, mostrou-se eficiente, pois permitiu a demarcação dos vasos neoformados, sua individualização e cálculo da área corneal vascularizada. Esta análise permitiu observar que a VC induzida pela MQ foi estatisticamente significativa $(\mathrm{p}<0,05)$ quando comparada aos olhos controles. Ressaltamos que em nenhum momento foram administrados fármacos anti-angiogênicos ${ }^{(23)} \mathrm{com}$ o intuito de reduzir a resposta inflamatória e conseqüente vascularização. A prevenção da rejeição aos enxertos corneais depende do uso de tais fármacos, inibindo assim a ativação dos linfócitos $T^{(19)}$. Sugerese a realização de estudos utilizando tais fármacos ${ }^{(24)}$, após implantação desta membrana na córnea, a fim de se investigar a resposta angiogênica, bem como se há minimização dos fenômenos clínicos aqui encontrados.

É possível que os fenômenos clínicos avaliados pudessem ter ocorrido de forma mais intensa se não fosse utilizada a buprenorfina $\left(\right.$ Tengesic $\left.^{\circledR}\right)$ que, neste caso, promoveu boa analgesia pós-operatória e conforto ocular ao animal. Salientase que o mesmo fora administrado por questões bioéticas; entendemos que tal procedimento deva ser empregado em trabalhos experimentais desta natureza.

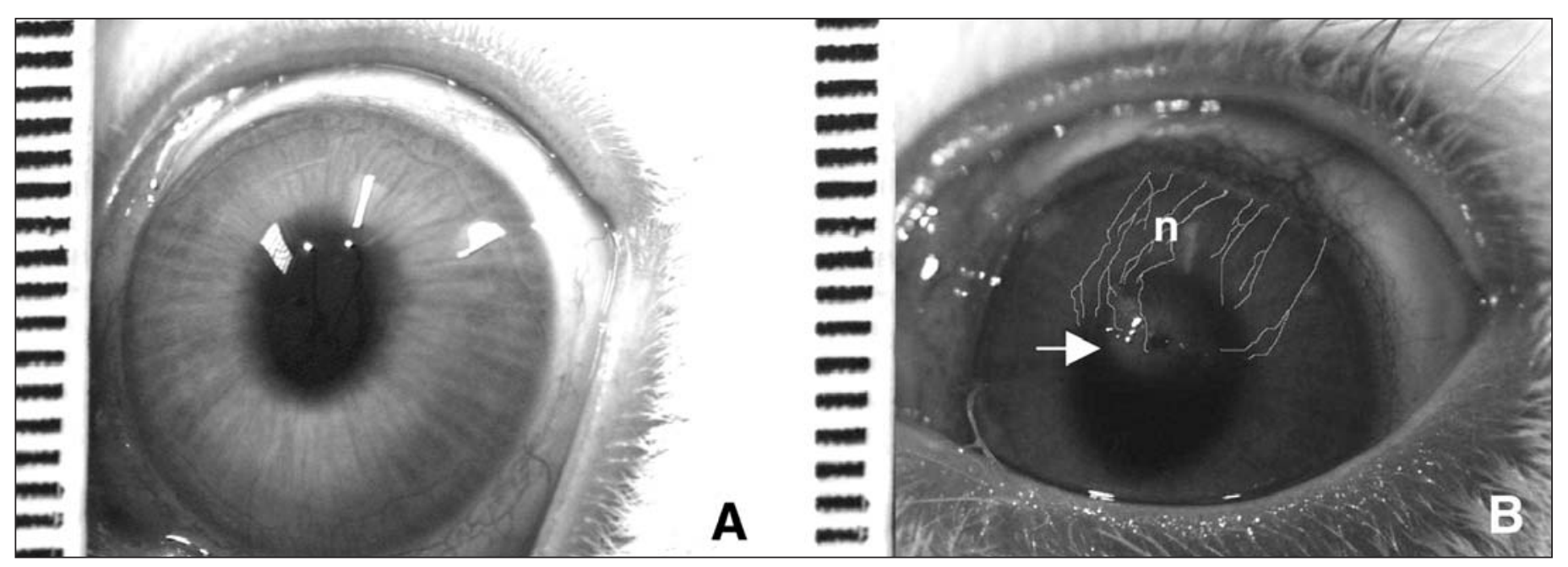

Figura 2 - Imagem fotográfica obtida do Sistema Analisador de Imagens LEICA QWIN $550^{\circledR}$ de olhos de coelhos que receberam ou não a membrana de quitosana, aos 30 dias de pós-operatório. Em A, olho controle. Em B, olho que recebeu MQ por enxertia interlamelar. Nota-se VC marcada (n) oriunda do limbo em direção ao implante (seta). Escala da régua em milímetros. 


\section{CONCLUSÕES}

Com base nos resultados obtidos e nas condições aqui concebidas pode-se concluir que:

1) $A M Q$ induziu angiogênese corneal quando aplicada à córnea de coelhos por enxertia interlamelar;

2) A VC induzida pela MQ foi observada a partir de 3 dias de pós-operatório, tornando-se intensa aos 15 dias, persistindo aos 30 dias de pós-operatório, porém de forma leve;

3) O Sistema Analisador de Imagens LEICA QWIN $550^{\circledR}$ mostrou-se eficiente na análise da área de VC;

4) Salienta-se a necessidade de estudos adicionais anatomopatológicos que possam estudar a biocompatibilidade desse enxerto, bem como, estudos por tempos mais prolongados.

\section{ABSTRACT}

Purpose: To evaluate corneal vascularization $(\mathrm{CV})$ induced by interlamellar graft chitosan membrane (CM) in rabbit cornea. Methodos: An interlamellar graft with a $0.25 \times 0.25 \mathrm{~cm} \mathrm{CM}$ fragment was performed in the left eye (treated eye). In the right eye, an estromal tunnel was done (control eye). The clinical evaluation was done at 1, 3, 7, 15 and 30 days postoperatively. CV analysis was after 30 days by the Images Analizator System LEICA QWIN-550 ${ }^{\circledR}$. Results: After 7 days, CV at $1.5 \pm 0.92 \mathrm{~mm}$ from the limbus in direction of the cornea axial area was observed. After 15 days CV increased $(4.75 \pm 3.19 \mathrm{~mm})$, remaining until day $30(4.25 \pm 4.06 \mathrm{~mm})$. The control eyes did not present any changes. There was a statistical differences of the vascularizated corneal areas between control and treated eyes from the $15^{\text {th }}$ to the $30^{\text {th }}$ postoperative day. Conclusions: The chitosan membrane induced corneal angiogenesis when applied to rabbit cornea through an interlamellar graft, which was maintained at low levels until 30 days postoperatively. Although further studies are necessary, the results found here demonstrated the usefulness of chitosan membrane in keratoplasties.

Keywords: Membranes, artificial; Cornea; Chitosan; Rabbits

\section{REFERÊNCIAS}

1. Gilger BC, Whitley D. Surgery of the cornea and sclera. In: Gelatt KN Veterinary ophthalmology. 3rd ed. Philadelphia: Lippincott Williams \& Willkins; 1999. p.675-700.

2. Vanathi M, Sharma N, Titiyal JS, Tandon R, Vajpayee RB. Tectonic grafts for corneal thinning and perforations. Cornea. 2002;21(8):792-7.
3. Barros PSM, Garcia JA, Laus JL, Ferreira A, Salles Gomes TL. Preserved equine amniotic membrane used in the repair of the cornea of the dog [abstract]. Invest Ophthalmol Vis Sci. 1995;36(4):S982. [Investigative Ophthalmology and Visual Science, Annual Meeting. Fort Lauderdale Florida May. 14-19, 1995].

4. Barros PSM, Laus JL, Morales A, Ferreira AL, Safatle AMV. Reparação experimental de lesões lamelares da esclera de cães com pericárdio xenólogo conservado. Arq Bras Oftalmol. 1996;59(5):462-6.

5. Andrade AL, Laus JL, Figueiredo F, Batista CM. The use of preserved equine renal capsule to repair lamellar corneal lesions in normal dogs. Vet Ophthalmol. 1999;2(2):79-82.

6. Rinaudo M, Domard A. Solution properties of chitosan. In: Skjak-Braek G, Anthonsen T, Sandford P. Chitin and chitosan. London: Elsevier Applied Science; 1989.p.71-86

7. Roberts GA. Chitin chemistry. Hampshire: Macmillan. 1992.

8. Li Q, Dunn ET, Grandmaison, EW, Goosen MFA. Application and properties of chitosan. J Bioact Compat Polym. 1992;7:370-97.

9. Sekiguchi S, Miura Y, Koneko H, Nisimura SI, Nishi N, Iwase M, Tokura S. Molecular weight dependency of antimicrobial activity by chitosan oligomers, In: Nishinari K, Doi E, editors. Food hydrocolloids: structures, properties, and functions. New York: Plenum Press. c1993.

10. Zheng LY, Zhu JF. Study on antimicrobial activity of chitosan with different molecular weights. Carbohydr Polym. 2003;54:527-30.

11. Harish Prashanth KV, Tharanathan RN. Depolymerized products of chitosan as potent inhibitors of tumor-induced angiogenesis. Biochim Biophys Acta. 2005; 1722(1):22-9.

12. Cwalina L, Mrukwa-Kominek E. [Corneal transplantation - immunological mechanisms of rejection episode]. Klin Oczna. 2004;106(4-5):686-90. Polish.

13. Vitova A, Filipec M, Zajicova A, Krulova M, Holan V. Prevention of corneal allograft rejection in a mouse model of high risk recipients. Br J Ophthalmol. 2004;88(10):1338-42.

14. Hegde S, Beauregard C, Mayhew E, Niederkorn JY. CD4(+) T-cell-mediated mechanisms of corneal allograft rejection: role of Fas-induced apoptosis. Transplantation. 2005;79(1):23-31.

15. Callanan D, Peeler J, Niederkorn JY. Characteristics of rejection of orthotopic corneal allografts in the rat. Transplantation. 1988;45(2):437-43.

16. Thoft RA, Friend J, Murphy HS. Ocular surface epithelium and corneal vascularization in rabbits. I. The role of wounding. Invest Ophthalmol Vis Sci. $1979 ; 18(1): 85-92$.

17. SAS Institute. SAS/STAT software: changes and enhancements through release 6.12. Cary: Statistical Analysis System Institute. 1997. 1167p.

18. Gimbrone MA Jr, Cotran RS, Leapman SB, Folkman J. Tumor growth and neovascularization: an experimental model using the rabbit cornea. J Natl Cancer Inst. 1974;52(2):413-27.

19. Gebhardt BM, Shi W. Experimental corneal allograft rejection. Immunol Res. 2002;25(1):1-26.

20. Lee CH, Singla A, Lee Y. Biomedical applications of collagen. Int J Pharm. 2001;221(1-2):1-22

21. Hunt TK, Knighton DR, Thakral KK, Goodson WH $3^{\text {rd }}$, Andrews WS. Studies on inflammation and wound healing: angiogenesis and collagen synthesis stimulated in vivo by resident and activated wound macrophages. Surgery. 1984;96(1):48-54.

22. Stoltz RA, Conners MS, Gerritsen ME, Abraham NG, Laniado-Schwartzman M. Direct stimulation of limbal microvessel endothelial cell proliferation and capillary formation in vitro by a corneal-derived eicosanoid. Am J Pathol. 1996;148(1):129-39.

23. Petri G. Influência da aplicação tópica da proteína ligante de cálcio MRP-14 na evolução da neovascularização induzida na córnea de ratos [tese]. São Paulo: Faculdade de Medicina Veterinária e Zootecnia. Universidade de São Paulo. 1997.

24. Slegers TP, van Rooijen N, van Rij G, van der Gaag R. Delayed graft rejection in pre-vascularised corneas after subconjunctival injection of clodronate liposomes. Curr Eye Res. 2000;20(4):322-4. 